\title{
A Dispersion Analysis for Difference Schemes: Tables of Generalized Airy Functions*
}

\author{
By R. C. Y. Chin and G. W. Hedstrom
}

Abstract. This paper contains graphs and tables of the function

$$
A i_{p, q}(\alpha, x)=\int_{-\infty}^{\infty}(2 \pi)^{-1} \exp \left\{i y p / p-\alpha y^{q} / q+i x y\right\} d y
$$

and its indefinite integral for $p=3,5,7$, for $q=2,4,6$, and for several values of $\alpha$ with $\alpha \geqslant 0$. It is shown how these tables should influence the choice of an artificial viscosity for a difference scheme for a linear hyperbolic equation.

1. Introduction. This paper consists primarily of graphs and tables of values of the generalized Airy function

$$
A i_{p, q}(\alpha, x)=(2 \pi)^{-1} \int_{-\infty}^{\infty} \exp \left\{i y^{p} / p-\alpha y^{q} / q+i x y\right\} d y
$$

and its integral

$$
A i i_{p, q}(\alpha, x)=(2 \pi i)^{-1} \int_{-\infty}^{\infty} y^{-1} \exp \left\{i y^{p} / p-\alpha y^{q} / q+i x y\right\} d y .
$$

Here, $\alpha \geqslant 0, x$ is real, and $p$ and $q$ are natural numbers such that $q$ is even and $p$ is odd, $p \geqslant 3$. The improper integral (1.2) is the Cauchy principal value. We computed these tables because the solution of a linear hyperbolic equation with constant coefficients behaves near a discontinuity like the integral $A i_{p, q}$. See [4] and [5]. Our tables show the magnitude and extent of the numerically induced oscillations.

Our results show that it is better to add an artificial viscosity to a nondissipative difference scheme than to use a difference scheme with the viscosity built in. The reason is that if $q<p$ as in the upstream-difference method, the viscosity becomes predominant as $t / h$ increases, and if $q>p$ as in the Lax-Wendroff method, the viscosity weakens as $t / h$ increases. On the other hand, for a nondissipative scheme with order of accuracy $p-1$, if the artificial viscosity is a discretization of

$$
Q=-(-1)^{q / 2} \delta h^{q(p-1) / p} q^{-1} \partial^{q} u / \partial x^{q}
$$

( $q$ even), then the amount of viscosity as measured by the parameter $\alpha$ in (1.1) and (1.2) is independent of $h$,

$$
\alpha=\delta t^{(p-q) / p} .
$$

We see that $\alpha$ gives a measure of the effective diffusive time scale. Note, though, that with the artificial viscosity (1.3) $\alpha$ is an increasing function of $t$ if $p>q$ and a decreasing function of $t$ if $p<q$.

Received September 30, 1976; revised January 23, 1978.

AMS (MOS) subject classifications (1970). Primary 65A05; Secondary 33A70, 65M15.

${ }^{*}$ This work was performed under the auspices of the U.S. Energy Research and Development Administration under contract number W-7405-Eng-48.

Copyright $\odot 1978$ American Mathematical Society 
The behavior of a high-order difference scheme near a discontinuity is as follows. Suppose we use a nondissipative difference scheme with order of accuracy $p-1$ and artificial viscosity (1.3). Then for fixed $t$ the oscillations near a discontinuity extend over a distance

$$
O\left(h^{(p-1) / p}\right)
$$

We see that there is a slight improvement as $p$ increases. Our tables show that as $p$ increases $(p=3,5,7)$ there is also a decrease in the amplitude of the oscillations. As we increase $q$, though, the damping near the wave front is decreased. In fact, it is only for $q=2$ that there is no overshoot.

Let us emphasize that we consider here only linear equations with linear artificial viscosity. The behavior of difference schemes for nonlinear equations with shocks is different. Nonlinear artificial viscosities of the form

$$
Q=-(-1)^{m} \delta h^{2 m(p-1) / p}(2 m)^{-1} D^{m}\left(|D u|^{r} D^{m} u\right)
$$

are in common use [9, p. 313], [7], where $D u$ denotes $\partial u / \partial x$. It is not usual, though, to use this particular power of $h$. The reason for using a nonlinear viscosity of the form (1.4) is that one wants the effect to be greatest when $|D u|$ is large. If $r$ is even, the computation is more efficient because we do not need to check sgn $(D u)$. Finally, let us remark that the clipping algorithm of Boris and Book [1] is very good for discontinuities of the sort $u=\operatorname{sgn}(x)$, but for $u=\operatorname{sgn}(x)-x$ it produces a staircase.

We have concentrated on the behavior of a difference scheme near a discontinuity of the solution of the differential equation. It is also of interest to know the behavior near a discontinuity of a derivative. If the discontinuity were in the derivative of order $r$, we would need to compute tables of the $(r+1)$-fold indefinite integral of the generalized Airy function $A i_{p, q}$.

Our graphs and tables are in the Appendix which appears in the microfiche section of this issue. In Section 2 we describe the graphs and tables and tell how we computed them. In Section 3 we show how the generalized Airy functions apply to various difference schemes.

2. Description of the Tables. In the Appendix are three sets of graphs with adjoining tables. The first set (Figures 1-17 and Tables 1-9) gives four local extrema of $A i_{p, q}(\alpha, x)$. The second set (Figures 18-23 and Tables 10-12) shows the function

$$
F_{q}(x)=(2 \pi)^{-1} \int_{-\infty}^{\infty} \exp \left\{-y^{q} / q+i x y\right\} d y
$$

and its integral. Finally, the third set (Figures 24-105 and Tables 13-53) consists of graphs and tables for the functions $A i_{p, q}$ and $A i i_{p, q}$.

The function $A i i_{p, q}(\alpha, x)$ is known [5] to oscillate about the value $-1 / 2$ for negative values of $x$. If $p=3$ and either $\alpha=0$ or $q=2$, we have

$$
\lim _{x \rightarrow \infty} \operatorname{Aii}_{p, q}(\alpha, x)=1 / 2
$$


monotonically. Otherwise, the function $\operatorname{Aii}_{p, q}(\alpha, x)$ oscillates about the value $1 / 2$ for positive values of $x$. The amplitudes of these oscillations are important in numerical analysis. The first set of graphs and tables shows the values for $p=3,5,7$ and $q=2$, 4,6 of $\operatorname{Aii}_{p, q}(\alpha, x)$ at the first three negative local extrema and at the first positive local maximum. Of the three negative local extrema the first is a local minimum and usually the absolute minimum, the second is a local maximum, and the third is a local minimum.

We point out some peculiarities. For $q=2$ and $p=5$ or $p=7$ the first two negative local extrema coalesce and disappear near $\alpha=3$ or $\alpha=3.2$, respectively. In fact, we see values of $\alpha$ for which the first negative local minimum is greater than $-1 / 2$. Similarly, for $q=2$ and $p=5$ the third and fourth negative extrema coalesce near $\alpha=4.8$, and for $q=2$ and $p=7$ the first and second positive local extrema coalesce near $\alpha=4.1$. For $p=3$ and $q=2$ there is no positive local maximum. For $p=3, q=4$, and $\alpha<0.3$ the first positive local maximum is too flat to compute accurately. For $p=3$ and $q=6$ the first two positive local extrema coalesce as $\alpha$ decreases to some value between 0.2 and 0.3 .

The second set of graphs and tables is labeled "P-term missing" and shows the function $F_{q}(x)$ defined by Eq. (2.1) and its integral, the Cauchy principal value of

$$
F i_{q}(x)=(2 \pi i)^{-1} \int_{-\infty}^{\infty} y^{-1} \exp \left\{-y^{q} / q+i x y\right\} d y .
$$

The importance of these functions is thid they show the limiting behavior of the generalized Airy functions for large viscosity,

$$
\lim _{\alpha \rightarrow \infty} \alpha^{1 / q} A i_{p, q}\left(\alpha, \alpha^{1 / q} x\right)=F_{q}(x), \quad \lim _{\alpha \rightarrow \infty} A i i_{p, q}\left(\alpha, \alpha^{1 / q} x\right)=F i_{q}(x) .
$$

For this reason we include the case $q=2$, even though $F_{2}$ is the Gaussian function

$$
F_{2}(x)=(2 \pi)^{-1 / 2} \exp \left\{-x^{2} / 2\right\}
$$

and $\mathrm{Fi}_{2}(x)$ differs from the error function by $1 / 2$. It is clear that $F_{q}$ is an even function, so that it would have been sufficient to plot and print its values for positive $x$. For ease of comparison with the generalized Airy functions we used for both $F_{q}$ and $A i_{p, q}$ a format appropriate for the Airy functions. We remark that since $\exp \left\{-y^{q} / q\right\}$ and $e^{i x y}$ are $G$-functions [8], it follows that $F_{q}(x)$ is also a $G$-function. In fact, it is not hard to show that in terms of the hypergeometric function ${ }_{0} F_{q-2}$ we have

$$
\begin{aligned}
F_{q}(x) & =\sum_{m=0}^{q-2} b_{m} x^{m}{ }_{0} F_{q-2}\left(; \delta_{m 1}, \delta_{m 2}, \ldots, \delta_{m, q-2} ;(-1)^{q / 2} x^{q} / q^{q-1}\right), \\
b_{m} & =0 \quad(m \text { odd }), \\
b_{m} & =(-1)^{m / 2} q^{(m+1) / q} \Gamma((m+1) / q) /(m ! q \pi) \quad(m \text { even }) .
\end{aligned}
$$

Here, $\delta_{m 1}, \delta_{m 2}, \ldots, \delta_{m, q-2}$ are the consecutive numbers $(m+2) / q,(m+3) / q$, $\ldots,(m+q) / q$ with $q / q$ omitted.

The first three negative local extrema for $\mathrm{Fi}_{4}$ and $\mathrm{Fi}_{6}$ are as follows 


\begin{tabular}{cc}
$\frac{x}{-2.44196790}$ & $F i_{4}(x)$ \\
-4.79724416 & -0.55220867 \\
-6.81358116 & -0.49384599 \\
$x$ & -0.50082421 \\
\hline-2.50081385 & $F i_{6}(x)$ \\
-4.93258336 & -0.57059517 \\
-7.23239945 & -0.47901803 \\
& -0.50617746
\end{tabular}

Hence, by (2.2) we have the limiting behavior of the first three local extrema of $A i_{p, q}$ for $q=4,6$. It is obvious that $F i_{2}$ is monotone.

The final set of graphs and tables is of values of $A i_{p, q}(\alpha, x)$ and $A i i_{p, q}(\alpha, x)$ for $p=3,5,7$ and $q=2,4,6$. They appear in the order $(p, q)=(3,2),(3,4),(3,6)$, $(5,2), \ldots$. For all values of $p$ and $q$ we give the generalized Airy function and its integral for $\alpha=0.5,1,2,4$. The case $\alpha=0$ is given only for $q=2$, because $A i_{p, q}(0, x)$ is independent of $q$. For $q=2$ and $p=5,7$ we include the case $\alpha=3$ in order to show the coalescence of the first two negative extrema.

We close this section with a description of the methods of computation. The derivative of order $r$ of the generalized Airy function may be written

$$
A i_{p, q}^{(r)}(\alpha, x)=\pi^{-1} \operatorname{Re} \int_{0}^{\infty}(i y)^{r} \exp \left\{i y^{p} / p-\alpha y^{q} / q+i x y\right\} d y,
$$

$r=-1,0,1, \ldots$ In order to make the exponential decay as $|y| \rightarrow \infty$, we replace the path of integration by the ray in the complex plane

$$
y=z e^{i \theta}, \quad 0<z<\infty, \theta=\pi /(2(p+q)) .
$$

For $r=-1$ we must add the contribution $2 \theta / \pi$ from the pole at the origin. For any given positive number $\epsilon$ it is easy to find an $R$ such that

$$
\pi^{-1}\left|\int_{R}^{\infty} z^{r} \exp \left\{i e^{i p \theta} z^{p} / p-\alpha e^{i q \theta} z^{q} / q+i x e^{i \theta} z\right\} d z\right|<\epsilon .
$$

We now use Romberg integration to compute the integral on the interval $0 \leqslant z \leqslant R$. In fact, the trapezoid rule for this integral is a finite complex Fourier series which is evaluated by the fast Fourier transform. The values of $A i_{p, q}$ and $A i i_{p, q}$ were computed by this method. The values of $F_{q}$ and $F i_{q}$ were computed in the same way but without the rotation of the path of integration. In order to find the local extrema of Aii $_{p, q}$, we used Newton's method, which requires evaluation of the integrals (2.3) for $r=-1$, 0,1 .

We checked our results as follows. First, it is easy to see that

$$
A i_{3,2}(\alpha, x)=\exp \{\alpha x / 2\} A i\left(x+\alpha^{2} / 4\right),
$$

where $A i$ is the usual Airy function. Second, the function $A i i_{p, q}$ is a solution of the ordinary differential equation

$$
(-1)^{(p-1) / 2} u^{(p)}-\alpha(-1)^{q / 2} u^{(q)}+x u^{\prime}=0,
$$

which may be solved numerically once we have evaluated the integral (2.3) at a starting 
value $x$ for $r=-1,0,1, \ldots, \max (p, q)-2$. The difficulty with this method is that for $\dot{q} \geqslant 6$ and for $p \geqslant 7$ (and also for $p=5$ if $x<0$ ) the differential equation (2.4) has a parasitic solution which dominates $A \ddot{i i}_{p, q}$ as $x$ increases and another parasitic solution which dominates $A i i_{p, q}$ as $x$ decreases. Hence, we must restart the computation fairly often. We found that it is better to start at a maximal value of $|x|$ and go toward the origin, using a stiff equation solver [6].

We make a remark regarding our method of computing values of the integral (2.3). The method is adequate for the values of $x$ we used, but for larger values of $|x|$ there would be roundoff error from cancellation. We would then need to use a contour which better approximates the optimal path through the saddle points [4], [5] .

3. Applications to Difference Schemes. We consider difference schemes for the equation

$$
u_{t}+u_{x}=0 \quad(-\infty<x<\infty, t>0)
$$

with $u(x, 0)=\operatorname{sgn}(x) / 2$. Our applications are based on the amplification function $[9$, p. 67] for the difference scheme. We assume that the difference scheme is stable. The amplification function has the form

$$
G(\xi)=\exp \{\lambda(-i \xi+i A(\xi)-B(\xi))\}
$$

where $A$ and $B$ are real, periodic functions such that

$$
\begin{array}{ll}
A(\xi)=c \xi^{p} / p+O\left(|\xi|^{p+2}\right) & (\xi \rightarrow 0), \\
B(\xi)=\epsilon \xi^{q} / q+O\left(\epsilon|\xi|^{q+2}\right) & (\xi \rightarrow 0) .
\end{array}
$$

Here, $p$ is odd, $q$ is even, $c \neq 0$, and $\lambda$ is the mesh ratio $\lambda=\Delta t / h, h=\Delta x$. It may happen that $B=0$, in which case the difference scheme is nondissipative. Otherwise, we have $\epsilon>0$.

It was shown in [4] that if $v_{k}^{n}$ denotes the solution of the difference scheme at the grid point $x=k h, t=n \Delta t$ with $v_{k}^{0}=\operatorname{sgn}(k h) / 2$, then near the wave front we have the asymptotic estimate

$$
\begin{aligned}
V_{k}^{n} & \sim A i i_{p, q}(\alpha, y) \\
\alpha & =\epsilon|c|^{-q / p}(\lambda t / h)^{(p-q) / p} \\
y & =\omega \operatorname{sgn}(c)|c|^{-1 / p}(t / h)^{(p-1) / p} .
\end{aligned}
$$

Here, $\omega$ is the scaled distance from the wave front $\omega=(x-t) / t$. It was shown in [4] that the region of validity of (3.2) if $q<p$ is

$$
\begin{aligned}
(\epsilon t / h)|c|^{-(p q+2) /\left(p^{2}-p\right)}|\omega|^{(q+2) /(p-1)} & \rightarrow 0, \\
|\omega||c|^{-1 / p}(t / h)^{(p-1) /(p+2)} & \rightarrow 0 .
\end{aligned}
$$

If $p<q$, the region of validity of (3.2) is [4]

$$
|\omega||c|^{-1 / q}(t / h)^{(q-1) /(q+2)} \rightarrow 0
$$

together with (3.3). Actually, the condition (3.3) was not needed in [4] because $\epsilon$ was taken to be constant there; it is not hard to show by the methods of [4] that (3.3) is needed if $\epsilon$ is allowed to vary. We remark that the numbers $p, q, c$, and $\epsilon$ for a difference scheme may be obtained from the Maclaurin series for $\log (G(\xi))$. 
If $p$ and $q$ are known, $c$ and $\epsilon$ may alternatively be obtained by a modification of the method used to find the local truncation error. This method is easier. One substitutes into the difference scheme a smooth solution $u$ of the equation

$$
u_{t}+u_{x}=(-1)^{(p-1) / 2} c p^{-1} h^{p-1} \partial^{p} u / \partial x^{p}-(-1)^{q / 2} \epsilon q^{-1} h^{q-1} \partial^{q} u / \partial x^{q}
$$

One then makes Taylor expansions, using Eq. (3.6) to eliminate derivatives with respect to $t$, and one chooses $c$ and $\epsilon$ so that the low-order terms cancel. We give an example in a moment. It is remarkable that this procedure is valid for a differential equation with a discontinuous solution, but it was shown in [4] and [5] that it is valid under conditions (3.3), (3.4) if $q<p$ and under conditions (3.3), (3.5) if $p<q$. Chin [2] has found the error of the approximation in a special case.

We now give some examples.

Example 1. Upstream differences. For the upstream-difference method

$$
v_{k}^{n+1}-v_{k}^{n}=\lambda\left(v_{k}^{n}-v_{k-1}^{n}\right)
$$

we know that $q=2$ and $p=3$. Upon substituting a smooth function $u$ into Eq. (3.7) and using Taylor expansions, we obtain

$$
\begin{aligned}
u_{t}+(\lambda h / 2) u_{t t}+ & \left(\lambda^{2} h^{2} / 6\right) u_{t t t}+O\left(h^{3}\right) \\
& =-u_{x}+(h / 2) u_{x x}-\left(h^{2} / 6\right) u_{x x x}+O\left(h^{3}\right) .
\end{aligned}
$$

In our case Eq. (3.6) becomes

$$
u_{t}=-u_{x}+(\epsilon h / 2) u_{x x}-\left(c h^{2} / 3\right) u_{x x x}
$$

so that

$$
u_{t t}=u_{x x}-\epsilon h u_{x x x}+O\left(h^{2}\right), \quad u_{t t t}=-u_{x x x}+O(h) .
$$

Consequently, the terms of (3.8) cancel to within $O\left(h^{3}\right)$ if and only if

$$
\epsilon=1-\lambda, \quad c=(1-\lambda)(1-2 \lambda) / 2 .
$$

Thus, if $\lambda \neq 1 / 2$, we have obtained $\epsilon$ and $c$ for the estimate (3.2) under conditions (3.3), (3.4). Note that $p \geqslant 5$ if $\lambda=1 / 2$.

Example 2. The Lax-Wendroff method. The Lax-Wendroff method [9, p. 331], when used for (3.1) has the amplification factor

$$
G(\xi)=1-i \lambda \sin \xi-\lambda^{2}(1-\cos \xi),
$$

so that

$$
\log G(\xi)=\lambda\left(i \xi+i\left(1-\lambda^{2}\right) 2^{-1} \xi^{3} / 3-\lambda\left(1-\lambda^{2}\right) 2^{-1} \xi^{4} / 4+O\left(\xi^{5}\right)\right)
$$

as $\xi \rightarrow 0$. Thus, we have (3.2) under conditions (3.3), (3.5) with $p=3, q=4$

$$
c=\left(1-\lambda^{2}\right) / 2, \quad \epsilon=\lambda\left(1-\lambda^{2}\right) / 2 .
$$

Example 3. Fromm's method. Fromm's method [3] when applied to (3.1) has the amplification factor

$$
G(\xi)=1-i(\lambda / 2) \sin \xi\left(1+e^{-i \xi}\right)-(\lambda / 2)\left(\lambda+(\lambda-2) e^{-i \xi}\right)(1-\cos \xi) .
$$

Consequently, we have 


$$
\log G(\xi)=\lambda\left(-i \xi+i c \xi^{3} / 3-\epsilon \xi^{4} / 4+O\left(\xi^{5}\right)\right)
$$

as $\xi \rightarrow 0$ with

$$
c=-(1-\lambda)(1-2 \lambda) / 4, \quad \epsilon=(1-\lambda)\left(1-\lambda+\lambda^{2}\right) / 2 .
$$

If $0<\lambda<1 / 2$ or $1 / 2<\lambda<1$, we have (3.2) under conditions (3.3), (3.5) with $p=3$ and $q=4$. If $\lambda=1 / 2$, we have to determine $p(p \geqslant 5)$ and $c$ by keeping higher order terms.

Example 4. The leapfrog scheme. The leapfrog scheme for (3.1) is

$$
v_{k}^{n+1}-v_{k}^{n-1}=\lambda\left(v_{k+1}^{n}-v_{k-1}^{n}\right)
$$

The solution to difference equation (3.9) has two waves, one with amplification factor

$$
G(\xi)=-i \lambda \sin \xi+\left(1-\lambda^{2} \sin ^{2} \xi\right)^{1 / 2},
$$

the other with amplification factor

$$
G_{2}(\xi)=-i \lambda \sin \xi-\left(1-\lambda^{2} \sin ^{2} \xi\right)^{1 / 2} .
$$

The wave generated by $G_{2}$ has a front with speed -1 , but it is easy to show that this wave has amplitude $O\left(h^{2}\right)$ if the starting values $v_{k}^{0}$ and $v_{k}^{1}$ are correct to within $O\left(h^{2}\right)$. Consequently, we focus our attention on $G_{1}$. It is clear that for $0<\lambda<1$ we have

$$
\left|G_{1}(\xi)\right|=1 \quad(-\infty<\xi<\infty)
$$

Hence, there is no $q$-term, or equivalently, $\alpha=0$ in (3.2). The Maclaurin series for $\log G_{1}(\xi)$ has the form

$$
\log G_{1}(\xi)=\lambda\left(-i \xi+i\left(1-\lambda^{2}\right) 2^{-1} \xi^{3} / 3+O\left(\xi^{5}\right)\right) .
$$

Consequently, we have $p=3$ and

$$
c=\left(1-\lambda^{2}\right) / 2 \text {. }
$$

Our asymptotic relation (3.2) is valid only if (3.4) holds, but because the difference scheme is not dissipative, the oscillations extend over a long distance. It is known [10] that the solution of (3.9) with initial data

$$
v_{k}^{0}=\operatorname{sgn}(k h) / 2, \quad v_{k}^{1}=\operatorname{sgn}(k h-\lambda h) / 2
$$

differs from the exact solution to $(3.1)$ by $O\left((h / t)^{1 / 2}\right)$ over every interval

$$
|x|<\theta t \quad(0<\theta<1) .
$$

In order to reduce this error, one usually adds an artificial viscosity. If this artificial viscosity is linear, the model equation (3.6) takes the form

$$
u_{t}+u_{x}=-\left(c h^{2} / 3\right) u_{x x x}-\epsilon(-1)^{q / 2} q^{-1} h^{q-1} \partial^{q} u / \partial x^{q} .
$$

One ordinarily chooses $q=2$ or $q=4$. If $q=2$ and $\epsilon=\delta h^{1 / 3}$ for a fixed constant $\delta$, then (3.2) takes the form

$$
\begin{aligned}
v_{k}^{n} & \sim A i i_{3,2}(\alpha, y), \\
\alpha & =\delta c^{-2 / 3}(\lambda t)^{1 / 3}, \\
y & =(x-t)(c t)^{-1 / 3} h^{2 / 3}
\end{aligned}
$$


with $c$ as in (3.10). Note that this choice of $\epsilon$ makes $\alpha$ independent of $h$. The artificial viscosity is a discretization of $\left(\delta h^{4 / 3} / 2\right) u_{x x}$. Similarly, if we want $q=4$ and

$$
v_{k}^{n} \sim A i i_{3,4}(\alpha, y)
$$

with $\alpha$ independent of $h$, we take $\epsilon=\delta h^{1 / 3}$. The artificial viscosity is then a discretization of $-\left(\delta h^{8 / 3} / 4\right) u_{x x x x}$.

Example 5. Fourth-order schemes. For a fourth-order, nondissipative scheme Eq. (3.2) takes the form

$$
v_{k}^{n} \sim A i_{5, q}(0, y)
$$

under condition (3.4) with $p=5$. Here,

$$
y=(x-t)(|c| t)^{-1 / 5} \operatorname{sgn}(c) h^{-4 / 5} .
$$

If we want positive $\alpha$ independent of $h$, we add to the difference scheme an artificial viscosity which is a difference approximation to one of the following

$$
\begin{aligned}
\delta h^{1.6} \partial^{2} u / \partial x^{2} & (q=2), \\
-\delta h^{3.2} \partial^{4} u / \partial x^{4} & (q=4), \\
\delta h^{4.8} \partial^{6} u / \partial x^{6} & (q=6), \ldots
\end{aligned}
$$

Other examples of model equations (3.6) are given in [11].

University of California

Lawrence Livermore Laboratory

P. O. Box 808

Livermore, California 94550

1. JAY P. BORIS \& DAVID L. BOOK, "Flux-corrected transport. I. SHASTA, A fluid transport algorithm that works," J. Computational Phys., v. 11, 1973, pp. 38-69.

2. RAYMOND C. Y. CHIN, "Dispersion and Gibbs phenomenon associated with difference approximations to initial boundary-value problems for hyperbolic equations," $J$. Computational Phys., v. 18, 1975, pp. 233-247.

3. JACOB E. FROMM, "A method for reducing dispersion in convective difference schemes," J. Computational Phys., v. 3, 1968, pp. 176-189.

4. G. W. HEDSTROM, "Models of difference schemes for $u_{t}+u_{x}=0$ by partial differential equations," Math. Comp., v. 29, 1975, pp. 969-977.

5. G. W. HEDSTROM, Generalized Airy Functions and Artificial Viscosities, Lawrence Livermore Laboratory report UCRL-78042.

6. A. C. HINDMARSH, GEAR: Ordinary Differential Equation System Solver, Lawrence Livermore Laboratory report UCID-30001, Rev. 1, A.C.C. No. 592, GEAR, Argonne Code Center, Building 221, Argonne National Laboratory, Argonne, Ill.

7. H.-O. KREISS \& J. OLIGER, "Comparison of accurate methods for the integration of hyperbolic equations," Tellus, v. 24, 1972, pp. 199-215. MR 47 \#7926.

8. YUDELL L. LUKE, The Special Functions and their Approximations, vol. 1, Academic Press, New York, 1969. MR 39 \#3039.

9. R. D. RICHTMEYER \& K. W. MORTON, Difference Methods for Initial Value Problems, 2nd ed., Interscience, New York, 1967. MR 36 \#3515.

10. S. I. SERDJUKOVA, "The oscillations that arise in numerical calculations of the discontinuous solutions of differential equations," $\check{Z}$. Vyčisl. Mat. $i$ Mat. Fiz., v. 11, 1971, pp. 411-424= U.S.S.R. Comput. Math. and Math Phys., v. 11, 1971, pp.140-154. MR 44 \#1248.

11. R. F. WARMING \& B. J. HYETT, "The modified equation approach to the stability and accuracy of finite-difference methods," J. Comput. Phys., v. 14, 1974, pp. 159-179. MR 49 \#4284. 